\title{
Numerical simulation and stability analysis of flow over a backward-facing step
}

\author{
Jiefang Liao ${ }^{1, a}$, Wei Zhang ${ }^{1, b}$ and Guoping Chen ${ }^{1, c}$ \\ ${ }^{1}$ School of Civil Engineering and Architecture, Southwest University of Science and Technology, \\ Mianyang, Sichuan, China, 621010 \\ akai-hou@139.com, 'bwell@swust.edu.cn, cguopingchen2002@163.com
}

Keywords: Flow over a backward-facing step, Numerical simulation, Suction, Blowing.

\begin{abstract}
Flow over a backward facing step has been one of important research field in fluid mechanics. By simulating the flow over a backward-facing step and analyzing the instability of fluid in different blowing and suction cases, the mechanism of structural damages and the principle of vortex shedding were studied. Using FLUENT software, stability change of flow over a backward-facing step were calculated and streamline pattern were drawn under the condition of without external excitation and blowing in the wall while sucking independently when $\mathrm{Re}=1000$. Furthermore, the result compared with that of Lambros Kaiktsis et al (2003).
\end{abstract}

\section{Introduction}

Flow over a backward-facing step is a typical flowing example of reattaching turbulent in fluid dynamics, which present the questions of flow over a bluff body in the case of a sudden expansion of transverse section. Due to the characteristics of numerous flow field structure and complex flow mechanism, researchers take the Flow over a backward-facing step as a typical turbulent flow case to study.

Armaly et al[1] makes numerous experimental research of flow over a backward-facing step, discussing the characteristics of flow field in different flow state. Comini[2, 3] takes the finite element method to study flow over a backward-facing step from the perspective of streamfunction-vorticity equation. Kaiktsis et al[4]'s research shows that $\mathrm{Re}=2500$ is the upper limit of flow stability. Later Barkley et al[5]'s research shows $\mathrm{Re} \approx 1000$ is the limit of flow stability for the Long-range flow. Lambros Kaiktsis and Peter A. Monkewitz[6] calculate the flow over a backward-facing step when $\mathrm{Re}=1000$, and inspect the suction and blowing's effect on stability. This thesis makes verification calculation in terms of the effect on the stability on blowing width $\delta$ and the distance $\Delta$ between blowing point and the wall.

\section{Meshing and numerical method}

The grid distribution after backward-facing step is as Fig. 1,

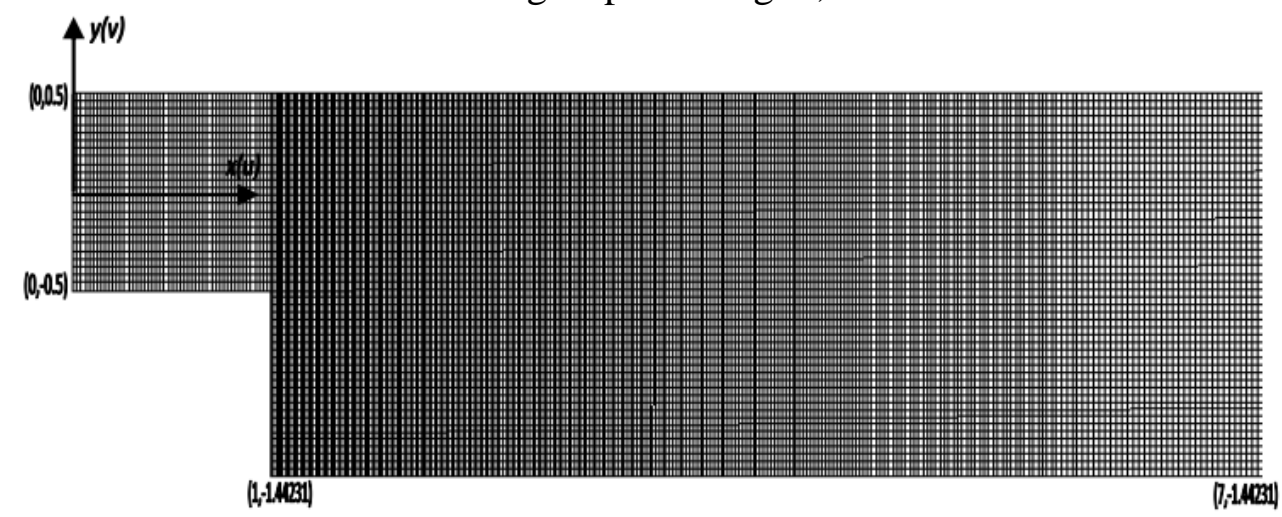

Fig.1 Grid distribution after backward-facing step. 
Taking GAMBIT software to meshing, this thesis takes evenly distributed quadrilateral meshes directly. The dense meshes are within $6 \mathrm{~m}$ near the step. The closer the meshes to the step, the denser they are, totally 49250 rectangular units. The paper takes FLUENT to solve flow field. As for Newtonian fluid with constant density, Navier-Stokes equation is taken. Nonmanipulated flow takes the Second Order Upwind discretion scheme and Coupled calculation. Meanwhile, manipulated flow takes QUICK Upwind discretion and SIMPLEC calculation.

\section{The basic model and boundary conditions}

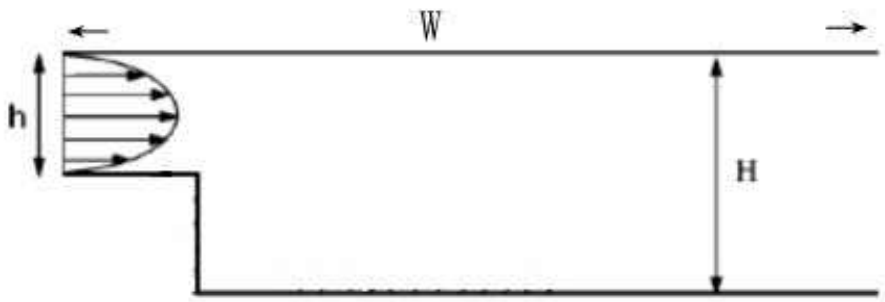

Fig.2 sketch of the nonmanipulated flow of backward-facing step.

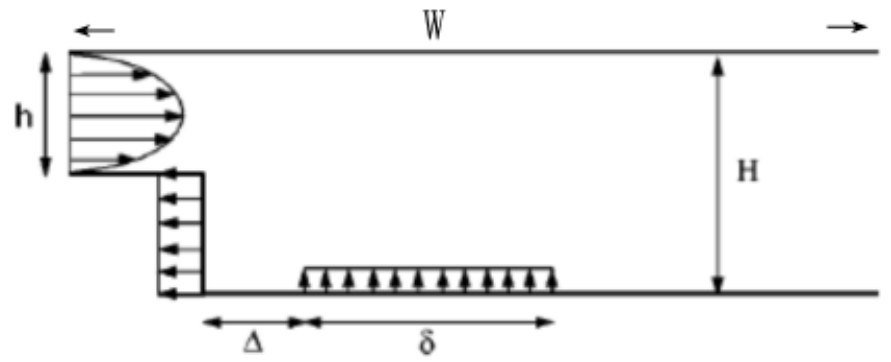

Fig.3 sketch of the manipulated flow of backward-facing step.

Referring to the literature of Lambros Kaiktsis and Peter A. Monkewitz[6], this calculation sets that $\mathrm{W}=35, \mathrm{H}=1.94231, \mathrm{~h}=1, \mathrm{Re}=1000$. The nonmanipulated flow does not take the consideration of external excitation, merely existing in inlet and outlet, showed as Figure. 2. The boundary conditions are as follows:

Inflow: $u=1-4 y^{2}, v=0$

Outflow: pressure outlet

Other wall and step surface are the solid walls.

In contrast, the manipulated flow adds some artificial factors. This thesis discusses blowing in the wall while sucking, showed as Figure. 3. The added boundary conditions are as follows,

Suction: $Q_{\text {suc }}=0.3 Q_{\text {inlet }}$

Blowing: $Q_{\text {blow }}=0.3 Q_{\text {inlet }}$

FLUENT cannot set the inflow boundary directly as that $u=1-4 y^{2}, v=0$, and defining the corresponding UDF is a must. Meanwhile, the UDF of $Q_{s u c}$ and $Q_{\text {blow }}$ is revised slightly based on the UDF of speed.

Reynolds number formula is as follows:

$$
\operatorname{Re}=\frac{4}{3} \frac{U_{\max } h}{v}
$$

From the boundary condition, $U_{\max }=1$. From the model structure, $\mathrm{h}=1$. When making numerical calculation, merely adjusting the fluid density and viscosity coefficient to ensure $\mathrm{Re}=1000$ is necessary.

\section{Results and analysis}


The steady-state of nonmanipulated flow. Boundary conditions and fluid parameters according to the definition of the above discourses, using the FLUENT software for numerical calculation, finally the following streamline pattern is obtained:

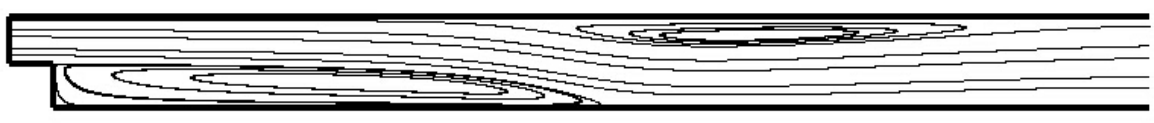

Fig.4 The steady-state streamline pattern of nonmanipulated flow.

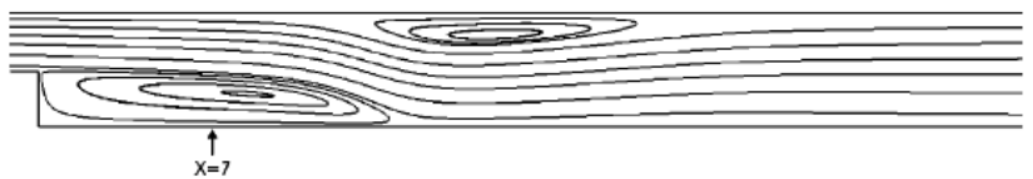

Fig.5 The steady-state streamline pattern of nonmanipulated flow (From Lambros Kaiktsis et al 2003), x/2.

From the part point of view, the flow field in and around the two vortex is unsteady. However, on the whole, the flow field remains stable. The result is basically the same with the streamline pattern (the X-scale is compressed by a factor of 2) in the paper of Lambros Kaiktsis et al (2003)[6].

The steady-state of manipulated flow. According to the paper of Lambros Kaiktsis et al (2003) ${ }^{[6]}$, the blowing width $\delta$ and the distance $\Delta$ between blowing point and the wall have effect on the stability of flowing. To verify this hypnosis, this paper sets $(\delta, \Delta)$ as $(2.0,1.2) 、(4.0,0.5) 、(2.0,0)$ 、 $(0.5,1.2) 、(0.8,0.5) 、(5.5,0)$, showed in Fig. 6.

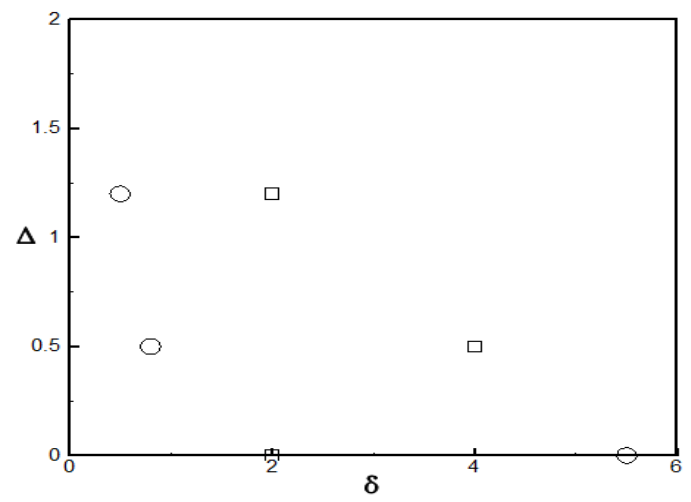

Fig.6 Distribution of calculation spot (unsteady, osteady)

Instantaneous streamline patterns of the six situations are as follows:

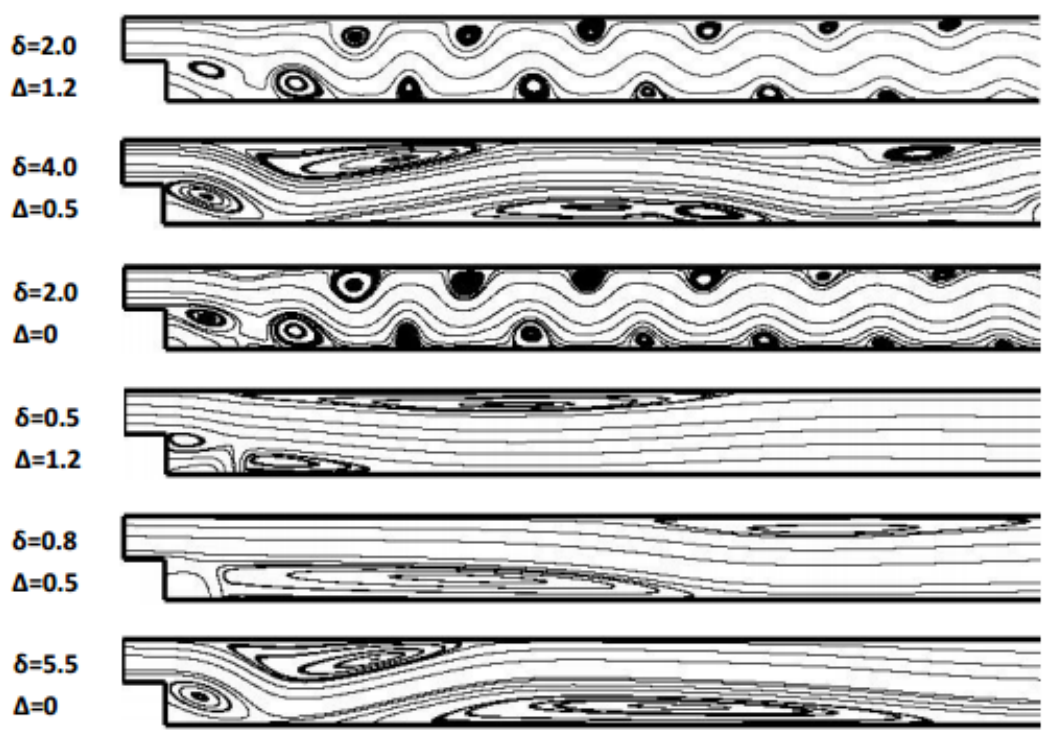

Fig.7 Instantaneous streamline patterns

From Fig. 7, the result shows that the different values of $\delta$ and $\Delta$ have effect on the stability of flow field. The flow field of unsteady flow presents to be periodical. In contrast, because of the enlargement of vortex, the stability is weaker than the nonmanipulated flow on the whole. The result is in accordance with the references. 


\section{Conclusions}

For the flow over a backward-facing step, this paper works out the stability change without the consideration of external excitation and sucking on the step while blowing on the wall, when $\mathrm{H} / \mathrm{h}=1.94231, \mathrm{Re}=1000$. The conclusion can be drawn that flow filed is generally remains steady, in the case of nonmanipulated flow, except the partial instability in the place of vortex. However, when sucking while blowing in the wall, the result is different. The flow field does not remain steady, changing with the change of parameter $\delta$ and $\Delta$.

Because of the different sets of specific parameter, part of the calculation results is in little discrepancy with the paper of Lambros Kaiktsis et al (2003), but are coincident on the whole. What effect blowing and suction in other wall have on the flow field can be further researched in the follow-up work.

\section{References}

[1] B.F.Armaly, F.Durst, J.C.F.Peireira and B.Schonung: Experimental and theoretical investigation of backward-facing step flow .J Fluid Mech. Vol.127 (1983), p. 473-496.

[2] G.Comini, M.Manzan and C.Nomino: Finite element solution of the stream-unction-vorticity equations for incompressible two-dimensional flows. Int. J. Num. Methods Fluids. Vol.19 (1994), p. 513-525.

[3] M.Manzan, G.Comini: Inflow and outflow boundary conditions in the finite element solution of the streamfunctions-vorticity equations. Commun. Num. Methods Engineering. Vol.11 (1995), p. 33-40.

[4] L.Kaiktsis, G.E.Karniadakis and S.A.Orszag: Unsteadiness and convective instabilities in two-dimensional flow over a backward-facing Step. J Fluid Mech. Vol.321 (1996), p.157.

[5] D.Barkley, M.G.M. Gomes, and R.D. Henderson: Three-dimensional instability in flow over a backward-facing step. J Fluid Mech. Vol.473 (2002), p.167.

[6] L. Kaiktsis, P.A. Monkewitz: Global destabilization of flow over a backward-facing step. Phys. Fluids. Vol.15 (12) (2003), p. 3647-3658. 